\title{
LncRNA LL22NC03-N14H11.1 promoted hepatocellular carcinoma progression through activating MAPK pathway to induce mitochondrial fission
}

Tingzhuang Yi ${ }^{1}$, Hongcheng Luo ${ }^{2}$, Fengxue Qin ${ }^{3}$, Qi Jiang ${ }^{1}$, Shougao He ${ }^{1}$, Tonghua Wang ${ }^{1}$, Jianwei Su${ }^{1}$, Sien Song ${ }^{1}$, Xiaoshan Qin', Yueqiu Qin', Xihan Zhou' ${ }^{1}$ and Zansong Huang ${ }^{1}$

\begin{abstract}
Involvement of long non-coding RNAs (IncRNAs) in hepatocarcinogenesis has been largely documented. Mitochondrial dynamics is identified to impact survival and metastasis in tumors including hepatocellular carcinoma ( $\mathrm{HCC}$ ), but the underlying mechanism remains poorly understood. This study planned to explore the regulation of InCRNA LL22NC03-N14H11.1 on HCC progression and mitochondrial fission. Dysregulated IncRNAs in HCC are identified through circlncRNAnet and GEPIA bioinformatics tools. Biological function of LL22NC03-N14H11.1 in HCC was detected by CCK-8 assay, flow cytometry analysis, transwell invasion, and wound healing assays. Molecular interactions were determined by RNA immunoprecipitation, RNA pull-down, and co-immunoprecipitation assays. Results showed that LL22NC03-N14H11.1 was upregulated in HCC tissues and cells. Functionally, LL22NC03-N14H11.1 contributed to cell proliferation, migration, invasion, and epithelial-to-mesenchymal transition (EMT) in HCC. Moreover, LL22NC03-N14H11.1 facilitated mitochondrial fission in HCC cells. Mechanistically, LL22NC03-N14H11.1 recruited Myb proto-oncogene (c-Myb) to repress the transcription of leucine zipper-like transcription regulator 1 (LZTR1), so as to inhibit LZTR1-mediated ubiquitination of H-RAS (G12V), leading to the activation of mitogen-activated protein kinase (MAPK) signaling and induction of p-DRP1 (Serine 616). In conclusion, this study firstly revealed that IncRNA LL22NC03N14H11.1 promoted HCC progression through activating H-RAS/MAPK pathway to induce mitochondrial fission, indicating LL22NC03-N14H11.1 as a novel potential biomarker for HCC treatment.
\end{abstract}

\section{Introduction}

Hepatocellular carcinoma (HCC) is the third most fatal malignancy globally, taking up $90 \%$ of primary liver cancer cases $^{1}$. Although during recent decades, surgery and

Correspondence: Yueqiu Qin (gxbsqyq@126.com) or

Xihan Zhou (zhxh1966@sohu.com) or Zansong Huang (1019846481@qq.com)

'Gastrointestinal Medicine, Affiliated Hospital of YouJiang Medical University

For Nationalities, Baise, Guangxi 533000, P. R. China

${ }^{2}$ Laboratory Medicine, Affiliated Hospital of YouJiang Medical University For

Nationalities, Baise, Guangxi 533000, P. R. China

Full list of author information is available at the end of the article

These authors contributed equally: Tingzhuang Yi, Hongcheng Luo, Fengxue Qin, Qi Jiang

Edited by G. Melino interventional therapies have been greatly advanced, survival of HCC patients is still unsatisfactory ${ }^{2}$, which is mainly attributed to easy metastasis and frequent recurrence $^{3}$. Therefore, further exploration of the precise mechanism behind HCC progression is urgently required.

Long non-coding RNAs (lncRNAs) are defined as a group of transcripts with a length over 200 nucleotides and lack of protein products ${ }^{4,5}$. Volumes of studies have offered convincing evidences that lncRNAs serve important roles in carcinogenesis of a wide range of cancer types ${ }^{6,7}$, including $\mathrm{HCC}^{8,9}$. Mechanistically, IncRNAs functioned through regulating target genes at epigenetic, transcriptional, and post-transcriptional levels ${ }^{10,11}$, and 
the mechanism varies depending on the cellular location of $\operatorname{lncRNAs}{ }^{12}$. Here, we firstly discovered a novel lncRNA LL22NC03-N14H11.1 located at chr22:16,154,073$16,154,766$ via bioinformatics analysis, and the results showed that LL22NC03-N14H11.1 was upregulated and had poor prognostic significance in HCC samples. Thus, we speculated that LL22NC03-N14H11.1 might participate in $\mathrm{HCC}$ progression and intended to probe into its role in $\mathrm{HCC}$.

The morphology of mitochondria is highly dynamic and varies constantly through fission and fusion, helping cells adjust their morphology to satisfy the variable need of cells and adapt to the cellular environment ${ }^{13}$. Studies have shown that mitochondrial fission is increased in tumor cells, and that inhibiting mitochondrial fission can impede proliferation and induce apoptosis in several cancer types, such as colon cancer and lung cancer ${ }^{14-16}$. Also, facilitated mitochondrial fission can aggravate cancer metastasis through promoting migration and invasion ${ }^{17,18}$. Notably, Huang et al. ${ }^{19}$ stated that mitochondrial fission was increased in $\mathrm{HCC}$ and promoted $\mathrm{HCC}$ cell survival and autophagy. However, whether LL22NC03-N14H11.1 played a part in HCC via its regulation on mitochondrial fission has never been explored.

Moreover, highly conserved dynamin-related GTPases are recognized as primary regulators of mitochondrial dynamics, and the typical one of which is dynamin-related protein $1(\mathrm{DRP} 1)^{13,20}$. Phosphorylation of DRP1 at Serine 616 (S616) is proved to be required for the activation and recruitment of DRP1 to the mitochondrial membrane to induce mitochondrial fission $^{21}$. For example, CDK1 phosphorylates Ser585 of rat DRP1, which is equivalent to human S616 during mitosis ${ }^{22}$. Protein disulfide isomerase (PDI1) facilitates DRP1 S616 phosphorylation and mitochondrial fission in CA1 neurons ${ }^{23}$. Multiple studies reveal that active ERK $1 / 2$ also contributes to the phosphorylation of DRP1 at $\mathrm{S} 16^{24}$. Importantly, DRP1 S616 phosphorylation by the activation of RAS/MAPK (mitogen-activated protein kinase) signaling is demonstrated to be oncogenic in cancer cells. For example, RAS (G12V)induced MAPK pathway led to DRP1 S616 phosphorylation and mitochondrial fission and tumor cell survival ${ }^{25}$. H-RAS (G12V) phosphorylated ERK1/2 and contributed to pancreatic cancer tumor growth through facilitating DRP1 S616 phosphorylation and mitochondrial fission ${ }^{26}$. To date, RAS/MAPK pathway has been largely reported to be involved in cell survival, apoptosis, and metastasis in cancers. However, the correlation of LL22NC03N14H11.1 with RAS/MAPK signaling has never been investigated yet.

Leucine zipper-like transcription regulator 1 (LZTR1) is a Golgi protein belonging to BTB-Kelch superfamily ${ }^{27}$, which is generally known to function through interacting with Cullin3 (CUL3)-based E3 ubiquitin ligases ${ }^{28,29}$.
LZTR1 has been reported to potentially participate in apoptosis and ubiquitination ${ }^{27,30,31}$. Several studies have revealed the tumor-suppressive role of LZTR1, and the germline and somatic mutations of LZTR1 in patients with glioblastoma and schwannomatosis ${ }^{32-34}$. Interestingly, it has been reported that LZTR1 is a conserved regulator of the ubiquitination of RAS family, leading to the degradation of both wild-type and mutant RAS members to inactivate MAPK pathway ${ }^{35}$. These findings indicated that LZTR1 might elicit its tumor-suppressive function through MAPK pathway, and that LZTR1 might be related to mitochondrial fission. However, the function and mechanism of LZTR1 in HCC and its association with mitochondrial fission have never been revealed.

Therefore, the present study aimed to uncover the role and mechanism of LL22NC03-N14H11.1 in HCC and its potential regulation on mitochondrial fission.

\section{Materials and methods \\ Clinical specimen collection}

Sixty-two patients diagnosed with $\mathrm{HCC}$ were recruited for collecting tumor and adjacent non-tumor tissue samples between 2013 and 2018, with the approval of Ethics Committee of Affiliated Hospital of YouJiang Medical University For Nationalities. Paired non-tumor tissues were at least $5 \mathrm{~cm}$ away from the tumor margin. Samples were frozen immediately in liquid nitrogen after surgical resection and stored at $-80^{\circ} \mathrm{C}$ until used for total RNA extraction. Patients treated with radiotherapy or chemotherapy before surgery were excluded, and all subjects had provided the written informed consent.

\section{Cell culture}

Normal liver cell line THLE-3, human renal epithelial cell line 293T, and HCC cell lines (Hep3B, SNU-449, LM3, Huh7 and SK-HEP-1) were acquired from the American Type Culture Collection (Manassas, VA, USA). In accordance with the protocol of Dulbecco's modified Eagle's medium (DMEM) (Gibco, Rockville, MD, USA) containing $1 \%$ penicillin/streptomycin (Gibco) and 10\% fetal bovine serum (FBS; Gibco), cells were grown routinely in $5 \% \mathrm{CO}_{2}$ at $37^{\circ} \mathrm{C}$. The medium was changed every 3 days, and cell passage was performed when the cells grew up to $80-90 \%$ confluence.

\section{Reagent and antibodies}

The commercially available mitochondrial division inhibitor, Mdivi-1, was from Sigma-Aldrich (Saint-Louis, MO, USA) and utilized for treating SK-HEP-1 and Huh7 cells, with DMSO (Sigma-Aldrich) as the control group. Translation inhibitor cycloheximide chase was also from Sigma-Aldrich for assessing protein stability. The primary antibodies against cleaved (c)-caspase-3 (ab2302, Abcam), total (t)-caspase-3 (ab13847, Abcam), c-caspase-6 
(ab2326, Abcam), t-caspase-6 (ab185645, Abcam), Bax (ab32503, Abcam), Bcl-2 (ab32124, Abcam), E-cadherin (ab40772, Abcam), N-cadherin (ab76057, Abcam), MMP2 (ab37150, Abcam), MMP7 (ab5706, Abcam), DRP1 (ab184247, Abcam), PDI1 (ab4644, Abcam), CDK1 (ab18, Abcam), H-RAS (G12V) (ab140571, Abcam), LZTR1 (ab106655, Abcam), c-Myb (ab109127, Abcam), and GAPDH (ab245356, Abcam), as well as secondary antibodies were all obtained from Abcam (Cambridge, MA, USA). Antibodies against p-DRP1 (S616) (\#3455S, Cell Signaling Technology, Danvers, MA, USA), p-ERK1/2 (\#4370S, Cell Signaling Technology), and ERK1/2 (\#4695S, Cell Signaling Technology) were obtained from Cell Signaling Technology (Danvers, MA, USA).

\section{Quantitative real-time PCR}

Total cellular RNA preparation and complementary DNA (cDNA) synthesis were separately conducted via TRIzol (Invitrogen, Carlsbad, CA, USA) and HighCapacity cDNA Reverse Transcription Kit (Thermo Fisher Scientific, Waltham, MA, USA). SYBR Green PCR Master Mix (Takara, Kyoto, Japan) was used for quantitative real-time PCR (RT-qPCR) analysis on ABI Prism 7900HT sequence detector (Applied Biosystems, Foster City, CA, USA). Calculation of relative gene expression was achieved using the $2^{-\Delta \Delta C T}$ method. GAPDH and U6 acted as endogenous controls to normalize the data. The specific PCR primers were presented as follows: LL22NC03-N14H11.1, forward primer, 5'-GAGTCTGGG GATCAGCATCG, reverse primer, $5^{\prime}$-TCCAGGGGGCT GGATAATGA-3'; LINC00152, forward primer, 5'-TACT GCTGAGAGACCCCCTC-3', reverse primer, 5'-TAGCC AAAGGTTGGAAGCCA-3'; RP11-620J15.3, forward primer, 5'-TGCGGGACACGTTATCACAA-3', reverse primer, 5'-TTCAGCTCTCACGTTCCACC-3'; H-RAS, forward primer, 5'-ACCTGTTCTGGAGGACGGTA-3', reverse primer, 5'-TCTCAACCACGCACCCAAAT-3'; LZTR1, forward primer, 5'-AGCGTGGACTTCGACCA TAG-3', reverse primer, $5^{\prime}$-GCCAGCGATGCACTGTTT C-3'; C-Myb, forward primer, 5'-GCGAAGGAGTTCTA AGGCGA-3' ${ }^{\prime}$, reverse primer, $5^{\prime}$-CCGGTAGCTGTCCTG TGAAG-3'; U1, forward primer, 5'-ACGGACGAAACAA TGACCGT- ${ }^{\prime}$, reverse primer, $5^{\prime}$-TTATTCACGCGTAC TCCGCA-3'; GAPDH, forward primer, 5'-CTGGGCTA CACTGAGCACC-3', reverse primer, $5^{\prime}$-AAGTGGTCGT TGAGGGCAATG-3'; U6, forward primer, 5'-GCAGACC GTTCGTCAACCTA-3', reverse primer, $5^{\prime}$-AATTCTGT TTGCGGTGCGTC-3'.

\section{Subcellular fractionation}

Nuclear and cytoplasmic fractions of SK-HEP-1 or Huh7 cells were separated utilizing the PARIS Kit (Thermo Fisher). Cell samples were first lysed in cell fractionation buffer. The pellet washed with TSE buffer
(10 mM Tris, 300 mMsucrose, $1 \mathrm{mM}$ EDTA, 0.1\% NP40 $\mathrm{PH} 7.5)$ at $4000 \mathrm{~g}$ for $5 \mathrm{~min}$ in a tabletop centrifuge at $4{ }^{\circ} \mathrm{C}$. The resulting supernatant discarded and the pellets were nucleus. The resulting supernatant from the first round of differential centrifugation was sedimented for $150 \mathrm{~min}$ at $14000 \mathrm{rpm}$ in a tabletop centrifuge. The resulting pellets were membranes and the supernatant were cytoplasm. RT-qPCR was followed to determine the location of LL22NC03-N14H11.1, with GAPDH or U6 as the cytoplasmic or nuclear control. The experiment was conducted in triplicate.

\section{Fluorescence in situ hybridization}

The RNA fluorescence in situ hybridization (FISH) probe for LL22NC03-N14H11.1 was designed and produced at RiboBio (Guangzhou, China). Cell samples were fixed in $4 \%$ formaldehyde for $15 \mathrm{~min}$ before washing in phosphate-buffered saline (PBS), and then cultured with pepsin and dehydrated. The air-dried cells were incubated with FISH probe in the hybridization buffer. Nuclei were counterstained with 4',6-diamidino-2-phenylindole (DAPI) and cells were observed using laser scanning confocal microscope (Zeiss, Jena, Germany). The experiment was conducted in triplicate.

\section{Transfection}

Specific short hairpin RNAs (shRNAs) against LL22NC03-N14H11.1 (sh-LL22NC03-N14H11.1\#1/2/3), LZTR1 (sh-LZTR1), ELF1 (sh-ELF1), AR (sh-AR) or cMyb (sh-c-Myb) and corresponding negative controls (shNC) were acquired from GeneChem (Shanghai, China). The interference sequences were presented as follows: shNC, 5'-CCGGGGATTCCTATCCCCTGTCAATCTCGA GTTTGACAGGGGAAACCAATCGTTTTTG-3'; sh-LL 22NC03-N14H11.1\#1， 5'-CCGGGCACTGGTATGGGC TGTCTATCTCGAGATAGACAGCCCATACCAGTG CTTTTTG-3'; sh-LL22NC03-N14H11.1\#2， 5'-CCGGG GAATCAGGCCTCCCAAATTTCTCGAGAAATTTGG GAGGCCTGATTCCTTTTTG-3'; sh-LL22NC03-N14H 11.1\#3, 5'-CCGGGATGGAGAAAGCACTCACCTACTC GAGTAGGTGAGTGCTTTCTCCATCTTTTTG-3'; shNC, 5'-CCGGGCTTAAGTTCAGAAGACTTGGACTCG AGCGAATTCAAGTCTTCTGAACCTTTTTTG-3', shLZTR1， 5'-CCGGTCAGGGTCTCGGGCTACAGGGCC TCGAGAGTCCCAGAGCCCGATGTCCCGTTTTTG-3'; sh-NC, 5'-CCGGTAGCTAGATAAAGTTATAGAATC TCGAGATCGATCTATTTCAATATCTTATTTTTG-3', sh-ELF1, 5'-CCGGTTCCTCCGTCTCCGTGAATACGCT CGAGAAGGAGGCAGAGGCACTTATGCTTTTTG-3'; sh-NC, 5'-CCGGAATTGTATAGAAACTATATCTGC TCGAGTTAACATATCTTTGATATAGACTTTTTG-3', sh-AR, 5'-CCGGAAGTTCATAATTCTCTGTCTGACT CGAGTTCAAGTATTAAGAGACAGACTTTTTTG-3'; sh-NC, 5'-CCGGACTTGAAAGCGCAAGGAATTAGCTC 
GAGTGAACTTTCGCGTTCCTTAATCTTTTTG-3', shc-Myb，5'-CCGGCCGCTCCCTAGGCGTGCGATTACTC GAGGGCGAGGGATCCGCACGCTAATTTTTTG-3'. The pcDNA3.1 vector targeting DRP1 or c-Myb, as well as the active H-Ras (G12V) expressed from retroviral vector LZRS, was applied for gene overexpression. These plasmids were transfected into SK-HEP-1 or Huh7 cells for $48 \mathrm{~h}$ in 6-well plates $\left(1 \times 10^{6}\right.$ cells/well), by Lipofectamine 3000 (Invitrogen, Carlsbad, CA, USA). The experiment was conducted in triplicate.

\section{CCK-8}

Transfected SK-HEP-1 or Huh7 cells were prepared in 96-well plates containing complete medium with $5 \times 10^{3}$ cells in each well, and $10 \mu \mathrm{L}$ of CCK-8 solution (Dojindo, Kumamoto, Japan) was added into each well for $2 \mathrm{~h}$ to detect cell viability. The optical density value at $450 \mathrm{~nm}$ was recorded at $0,24,48,72$, and $96 \mathrm{~h}$ using a microplate reader (Dynex Technologies, West Sussex, UK). The experiment was conducted in triplicate.

\section{Colony formation}

Transfected SK-HEP-1 or Huh7 cells at the logarithmic growth phase were trypsinized, harvested, and planted into 6-well plates for 2 weeks, at the density of 500 cells per well. After washing in PBS, colonies containing more than 50 cells were fixed in methanol (Sigma-Aldrich), dyed in crystal violet (Sigma-Aldrich), and eventually counted manually. The experiment was conducted in triplicate.

\section{Flow cytometry of apoptosis}

Annexin-V-FITC/Propidium Iodide (PI) Apoptosis Detection Kit (BD Biosciences, Franklin Lakes, NJ, USA) was obtained for flow cytometry analysis. A total of $1 \times$ $10^{6}$ cells of SK-HEP-1 or Huh7 were collected after transfection, and plated into 6-well plates for treatment in $100 \mu \mathrm{L}$ of $1 \times$ Binding Buffer containing $5 \mu \mathrm{L}$ of PI and $5 \mu \mathrm{L}$ of Annexin-V-FITC. After culturing in a dark room for $15 \mathrm{~min}$, cell apoptosis of transfected SK-HEP-1 or Huh7 cells was analyzed by FACScan (BD Biosciences) and FlowJo V10 software (Tree Star, Ashland, OR, USA). The experiment was conducted in triplicate.

\section{Western blotting}

Total cellular protein samples were prepared using RIPA lysis buffer and subjected to sodium dodecyl sulfate (SDS)-polyacrylamide gel, following moving onto PVDF membranes (Bio-Rad Laboratories, Hercules, CA, USA). After blocking with 5\% nonfat milk, membranes were cultivated with primary antibodies at $4{ }^{\circ} \mathrm{C}$ all night. Following washing in Tris-buffered saline/Tween-20 (TBST), membranes were probed with secondary antibodies at room temperature for $2 \mathrm{~h}$. The band density was analyzed with application of the ECL luminous liquid (Pierce, Rockford, IL, USA) and quantified via the ImageJ software (National Institutes of Health, USA). The experiment was conducted in triplicate.

\section{Invasion assay}

Cell invasion was explored by 24-well Transwell chambers (8-mm pore size; Corning Incorporated, Big Flats, NY, USA) pre-coated with Matrigel (BD Biosciences). The upper chamber was added with $2 \times 10^{4}$ transfected cells in serum-free medium, while lower chamber was filled with complete culture medium containing 10\% FBS. Invaded cells on the bottom of membrane were fixed by $4 \%$ formaldehyde after $24 \mathrm{~h}$, stained with crystal violet, and counted under the optical microscope (Thermo Fisher). The experiment was conducted in triplicate.

\section{Wound healing}

Transfected SK-HEP-1 or Huh7 cells $\left(1 \times 10^{6}\right)$ were seeded into 6-well plates and cultured until cells reached 80-90\% confluence. The following day, cells were treated with a $200 \mu \mathrm{L}$ filter tip for creating the wound area, followed by $24 \mathrm{~h}$ of incubation at $37^{\circ} \mathrm{C}$ in serum-free medium. The distance of wound healing was photographed under a microscope (Olympus, Tokyo, Japan) at 0 or $24 \mathrm{~h}$. The experiment was conducted in triplicate.

\section{Immunofluorescence}

Transfected SK-HEP-1 or Huh7 cells in PBS (SigmaAldrich) were plated onto culture slides for $24 \mathrm{~h}$, incubated in $1 \%$ paraformaldehyde (Sigma-Aldrich) for $10 \mathrm{~min}$, permeabilized in methanol, and blocked with $0.8 \%$ bovine serum albumin (Sigma-Aldrich) for $10 \mathrm{~min}$. After incubation all night with primary antibodies against E-cadherin and $\mathrm{N}$-cadherin, secondary antibodies were added for $2 \mathrm{~h}$. Coverslips were mounted on glass slides after staining with DAPI (Sigma-Aldrich) for $10 \mathrm{~min}$, and were finally examined using the TE2000-U microscope (Nikon, Tokyo, Japan). The experiment was conducted in triplicate.

\section{Mitochondrial staining and mitochondrial fission analysis}

SK-HEP-1 or Huh7 cells were transplanted onto coverslips and transfected. Cells were centrifuged for $5 \mathrm{~min}$ at room temperature for treatment with $0.1 \mu \mathrm{M}$ of MitoTracker Red CMXRos (Molecular Probes, Thermo Fisher) for $30 \mathrm{~min}$. After washing, images were taken using laser scanning confocal microscope. The experiment was conducted in triplicate.

\section{Co-immunoprecipitation}

Transfected cells were reaped from immunoprecipitation lysis buffer, centrifuged, and then cell lysates were incubated overnight with indicated antibodies at a constant speed at $4{ }^{\circ} \mathrm{C}$. Normal immunoglobulin G (IgG) was seen as a negative 
control. Following culturing with protein A-sepharose beads, the antigen-antibody mixture was washed three times with IP lysis buffer, eluted, and subjected to immunoblotting (Western blot). The experiment was conducted in triplicate.

\section{Ubiquitination assay}

Transfected cells were incubated in hot lysis buffer containing 1\% SDS and $10 \mathrm{mM}$ of $N$-ethylmaleimide (Sigma-Aldrich) and boiled at $100^{\circ} \mathrm{C}$ for $10 \mathrm{~min}$. Cell lysates were diluted in SDS-free cell lysis buffer and mixed with anti-Flag-M2 agarose and assessed by western blotting. The experiment was conducted in triplicate.

\section{Luciferase reporter assay}

The wild-type or mutant sequences of c-Myb in LZTR1 promoter were sub-cloned into pGL3-basic vector (Promega, Madison, WI, USA) and co-transfected into 293T cells with pcDNA3.1/c-Myb or pcDNA3.1. The pGL3-LZTR1 promoter vector was co-transfected into SKHEP-1 or Huh7 cells with sh-LL22NC03-N14H11.1\#1/2 or sh-NC. Luciferase activities were studied with DualLuciferase reporter assay system (Promega), using Renilla luciferase as the internal control. The experiment was conducted in triplicate.

\section{RNA pull-down assay}

RNA pull-down assay was studied by Pierce Magnetic RNA-Protein Pull-Down Kit (Thermo Fisher Scientific, Waltham, MA, USA). Cell protein lysates of SK-HEP-1 or Huh7 were acquired by using RIPA lysis buffer, and then incubated with biotinylated RNA probes (LL22NC03N14H11.1 biotin or LL22NC03-N14H11.1 no biotin). The streptavidin-coated magnetic beads (Invitrogen) were added to capture the RNA-protein mixture. Western blot was applied for analysis of the enrichment of proteins pulled down in indicated groups. The experiment was conducted in triplicate.

\section{RNA immunoprecipitation}

Magna RIP ${ }^{\mathrm{TM}}$ RNA-Binding Protein Immunoprecipitation Kit was bought from Millipore (California, USA). Cell lysates were prepared using RNA immunoprecipitation (RIP) lysis buffer, and then cultivated with RIP buffer and magnetic beads conjugated to anti-c-Myb antibody, with anti-IgG antibody as a negative control. Besides, the interaction between U1 and SNRNP70 was used as a positive control for such assays. After adding proteinase $\mathrm{K}$, precipitates were assayed by RT-qPCR. The experiment was conducted in triplicate.

\section{Chromatin immunoprecipitation}

Chromatin immunoprecipitation (ChIP) assay was undertaken with the application of EZ ChIP ${ }^{\mathrm{TM}}$ Chromatin Immunoprecipitation Kit (Millipore), following the standard method. Cells were treated in formaldehyde for 10 min for generating the DNA-protein cross-links. Then, cell lysates were sonicated to acquire chromatin fragments of 200-300 bp and immunoprecipitated with antibodies against c-Myb and control IgG. RT-qPCR was followed for the retrieved precipitated chromatin DNA. The experiment was conducted in triplicate.

\section{Tumor xenograft model}

Four-week-old male BALB/c nude mice were purchased from Shi Laike Company (Shanghi, China) and maintained in SPF-grade, pathogen-free animal lab. Tumor xenograft assay was performed via injecting nude mice subcutaneously with $1 \times 10^{6}$ SK-HEP-1 cells transfected with sh-LL22NC03-N14H11.1\#1, shLL22NC03-N14H11.1\#1 + sh-LZTR1, or sh-NC (mice were randomly divided into three groups). Tumor volume was recorded every 4 days. Four weeks later, mice were killed by cervical dislocation and tumors were weighted. Animal studies were approved by the Animal Ethics Committee of Affiliated Hospital of YouJiang Medical University For Nationalities.

\section{TUNEL assay}

To perform terminal deoxynucleotidyl transferase dUTP nick-end labeling (TUNEL) assay in xenograft tissues, One-Step TUNEL Apoptosis Assay Kit was commercially acquired and used as instructed (Beyotime, Shanghai, China). After treatment with proteinase $\mathrm{K}$ for $15 \mathrm{~min}$ at room temperature, sections were fixed in $4 \%$ paraformaldehyde for $1 \mathrm{~h}$ and permeabilized with $0.1 \%$ Triton-X100 for $2 \mathrm{~min}$, followed by incubation with TUNEL Assay Kit. The fluorescence microscope (Leica, Heerbrugg, Canton of St. Gallen, Switzerland) was utilized for observing the TUNEL-stained and DAPI-stained sections. The apoptotic nuclei were determined as the TUNEL and DAPI-positive nuclei located within tumor tissues. The experiment was conducted in triplicate.

\section{Immunohistochemistry}

The tumor tissues were acquired from xenograft assay and then fixed in $4 \%$ paraformaldehyde, dehydrated, and embedded in paraffin. Four-millimeter-thick sections were cut from paraffin-embedded xenograft tissues and deparaffinized, followed by cultivation with antibodies against Ki67 (Abcam), proliferating cell nuclear antigen (PCNA) (Abcam), E-cadherin and $\mathrm{N}$-cadherin overnight at $4{ }^{\circ} \mathrm{C}$, and with biotinylated second antibody for $30 \mathrm{~min}$ at $37^{\circ} \mathrm{C}$. The experiment was conducted in triplicate.

\section{Hematoxylin and eosin staining}

Tissues from xenograft model were immobilized for $24 \mathrm{~h}$ utilizing $4 \%$ formaldehyde and paraffin-embedded, followed by sectioned and stained with hematoxylin and 


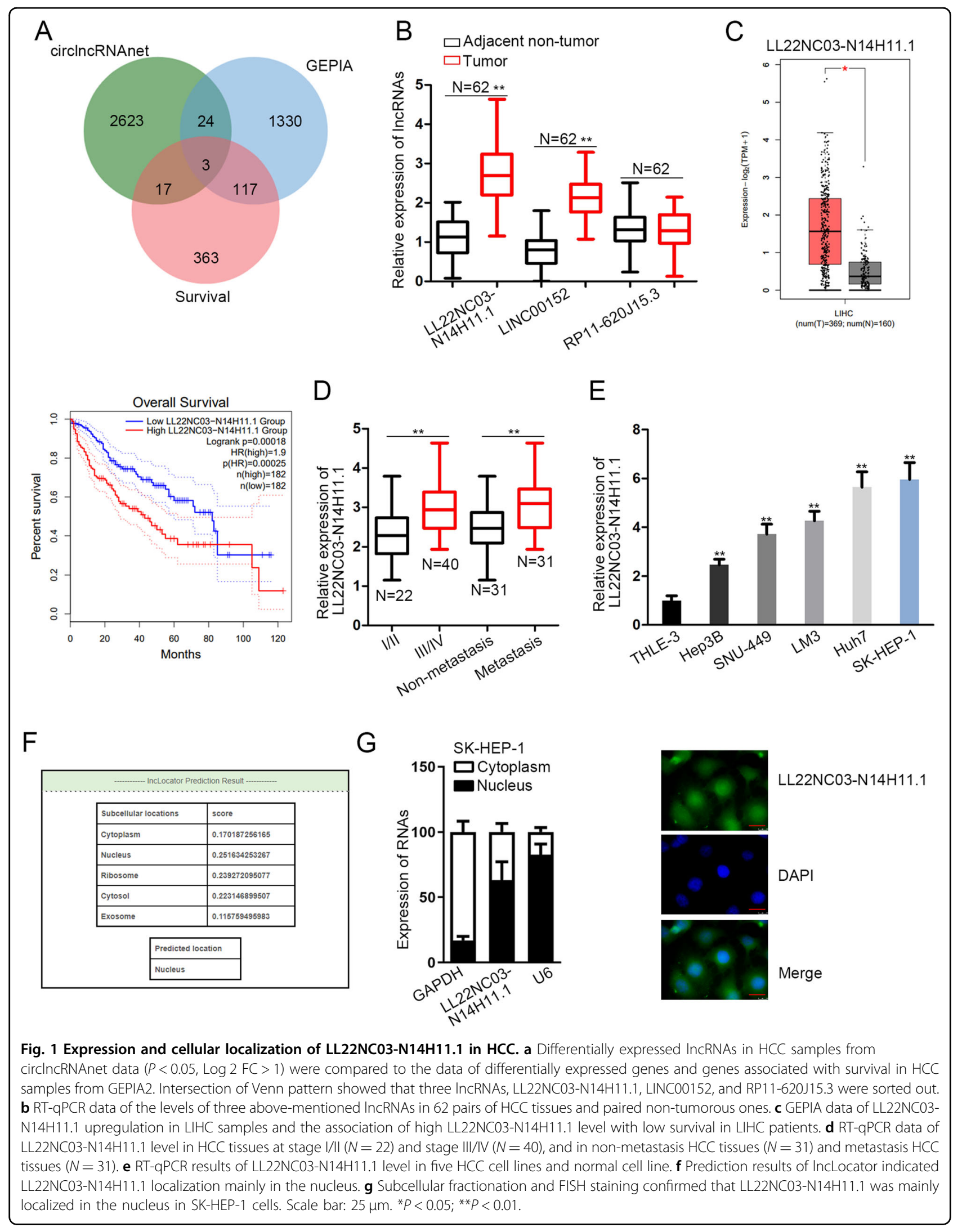


eosin (HE) (Sigma-Aldrich). The optical microscope (Nikon) was applied for analyzing. The experiment was conducted in triplicate.

\section{Statistical analysis}

Data (in line with normal distribution) from assays conducted in triplicate were listed as mean \pm SD. Statistical analysis was conducted by SPSS V.19.0 (SPSS, Chicago, IL, USA) or Prism 6 (GraphPad Software, San Diego, CA, USA). The overall survival was plotted via the Kaplan-Meier method and compared by log-rank test. Gene expression correlation was analyzed with Pearson's method. Student's $t$ test or analysis of variance was appropriately adopted for significant differences in groups, with $P<0.05$ as threshold.

\section{Results}

LL22NC03-N14H11.1 was upregulated in HCC and mainly located in the nucleus

First, to identify the dysregulated lncRNAs in HCC, we browsed circlncRNAnet (http://120.126.1.61/circlnc/ circlncRNAnet/lncRNA_TCGA/index.php) and identified 2667 upregulated lncRNAs in HCC samples $(P<0.05$, $\log 2$ fold change $(\mathrm{FC})>1$ ). Then, we analyzed these lncRNAs in the GEPIA database (Gene Expression Profiling Interactive Analysis) (http://gepia2.cancer-pku.cn/ \#index), finding that three lncRNAs, LL22NC03N14H11.1, LINC00152, and RP11-620J15.3, presented significant upregulation in liver HCC (LIHC) samples and had potential prognostic values in LIHC patients (Fig. 1a). Therefore, we examined the expression of the three lncRNAs in HCC tissues collected from our institute. RTqPCR data revealed that both LL22NC03-N14H11.1 and LINC00152 were highly expressed in 62 HCC samples vs. the matched adjacent non-tumor samples, and the upregulation of LL22NC03-N14H11.1 was more significant than LINC00152 (Fig. 1b). Since LINC00152 has already been reported as an oncogene in HCC by several previous studies $^{36-38}$, we focused on the role of LL22NC03N14H11.1 in HCC here. The significant elevation of LL22NC03-N14H11.1 in LIHC samples $(n=369)$ vs. normal tissues $(n=160)$ and the positive association of high LL22NC03-N14H11.1 level with low overall survival in LIHC patients $(p=0.00018)$ according to the GEPIA database were shown in Fig. 1c. Besides, we verified that LL22NC03-N14H11.1 was highly expressed in HCC patients at advanced clinical stages (stage III/IV, $n=40$ ) compared with primary stages (stage I/II, $n=22$ ) (Fig. 1d). Also, LL22NC03-N14H11.1 level was higher in metastatic HCC patients $(n=31)$ than non-metastatic HCC patients $(n=31)$ (Fig. 1d). These data indicated that LL22NC03-N14H11.1 potentially participated in tumor growth and metastasis in HCC. Moreover, upregulation of LL22NC03-N14H11.1 was validated in five HCC cell lines
(Hep3B, SNU-449, LM3, Huh7, and SK-HEP-1) vs. the normal THLE-3 cells, and SK-HEP-1 and Huh7 cells presented the highest LL22NC03-N14H11.1 level (Fig. 1e). In addition, through lncLocator (http://www. csbio.sjtu.edu.cn/bioinf/lncLocator/), we found that LL22NC03-N14H11.1 was mainly expressed in the nucleus (score $=0.252$ ) (Fig. 1f). Subcellular fractionation and FISH staining also confirmed that LL22NC03N14H11.1 was expressed in the nucleus more than in the cytoplasm of SK-HEP-1 cells (Fig. 1g). In collection, LL22NC03-N14H11.1 was upregulated in HCC and was mainly expressed in the nucleus of HCC cells.

\section{LL22NC03-N14H11.1 aggravated proliferation, prevented apoptosis, drove migration, invasion, and EMT in HCC in vitro}

Next, we detected the biological role of LL22NC03$\mathrm{N} 14 \mathrm{H} 11.1$ in $\mathrm{HCC}$ through in vitro loss-of-function assays conducted in Huh7 and SK-HEP-1 cells expressing high LL22NC03-N14H11.1 level. Successfully, RTqPCR data confirmed the overt silence of LL22NC03N14H11.1 by three shRNAs in both the HCC cell lines, and sh-LL22NC03-N14H11.1\#1/2 showed higher knockdown efficiency (Fig. 2a). Therefore, cells transfected with sh-LL22NC03-N14H11.1\#1/2 were applied for subsequent experiments. Consequently, silence of LL22NC03-N14H11.1 attenuated the viability and colony formation ability of two HCC cell lines (Fig. 2b, c). According to flow cytometry analysis, ratio of apoptotic HCC cells increased under the silence of LL22NC03N14H11.1 (Fig. 2d). Concordantly, levels of pro-apoptotic genes (cleaved-caspase-3, cleaved-caspase-6, and Bax) increased, whereas the level of anti-apoptotic gene $(B c l-2)$ decreased responding to LL22NC03-N14H11.1 silence in HCC cells, with total caspase- 3 and caspase- 6 unchanged (Fig. 2e), further suggesting that LL22NC03-N14H11.1 depletion increased apoptosis in HCC cells. Besides, the effect of LL22NC03-N14H11.1 on metastasis of HCC cells was assessed. We observed the retarded invasion of $\mathrm{HCC}$ cells under LL22NC03-N14H11.1 silence through a transwell system (Fig. 2f). Scratch wound assay depicted that LL22NC03-N14H11.1 knockdown inhibited migration of HCC cells (Fig. 2g). Additionally, EMT markers were examined in HCC cells. Western blot demonstrated that under the knockdown of LL22NC03-N14H11.1, Ecadherin level increased, whereas $\mathrm{N}$-cadherin, MMP2, and MMP7 levels decreased in HCC cells (Fig. 2h). Meanwhile, IF analysis indicated the augmented E-cadherin level and declined N-cadherin staining in SK-HEP-1 and Huh7 cells in response to LL22NC03-N14H11.1 inhibition (Fig. 2i). These data suggested that LL22NC03N14H11.1 silence hindered EMT progression in HCC cells. Meanwhile, we also implemented gain-of-function assays in Hep3B and SNU-499 cells. As expected, after 


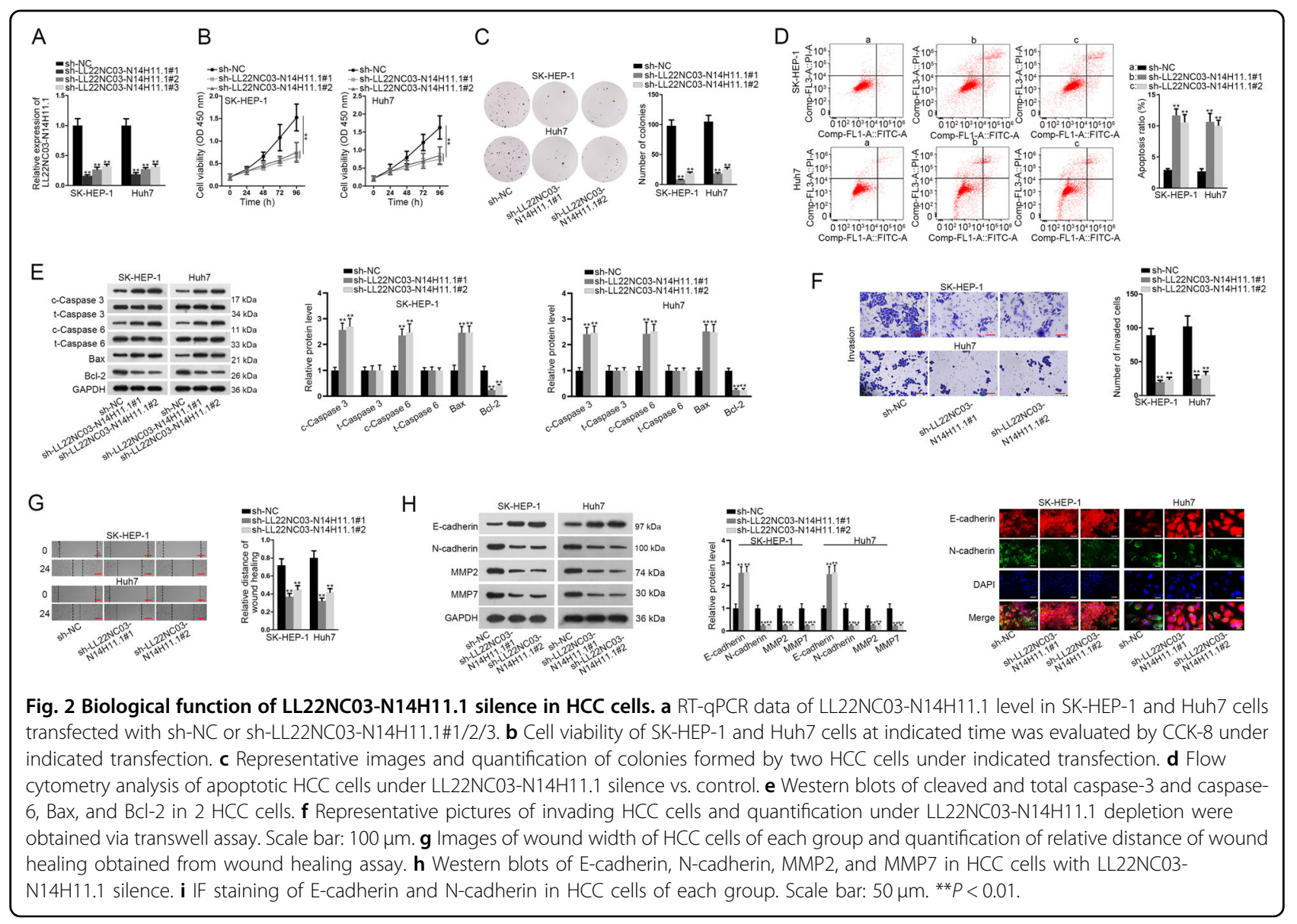

elevating LL22NC03-N14H11.1 expression in these two cells, the viability and proliferative ability were greatly strengthened (Supplementary Fig. S1A-C). Also, enhanced level of LL22NC03-N14H11.1 led to promoted motility in Hep3B and SNU-499 cells (Supplementary Fig. S1D, E). Besides, we observed facilitated EMT process in LL22NC03-N14H11.1-upregulated HCC cells (Supplementary Fig. S1F-G). Together, these data indicated that LL22NC03-N14H11.1 aggravated proliferation, migration, and invasion, as well as EMT process in HCC in vitro.

\section{LL22NC03-N14H11.1 promoted HCC progression through inducing p-DRP1 (S616) and facilitating mitochondrial fission}

A recent study reported that facilitated mitochondrial fission improved the survival and impeded apoptosis in HCC cells ${ }^{19}$. Moreover, studies have shown that inactivation of mitochondrial fission constrained cell migration and invasion in cancer ${ }^{17,18}$. Therefore, we wondered whether LL22NC03-N14H11.1 could regulate mitochondrial fission in $\mathrm{HCC}$ cells. MitoTracker Red staining analysis demonstrated that the mitochondrial elements were elongated and interconnected under LL22NC03-
N14H11.1 knockdown or the treatment of Midiv-1, the selective inhibitor of DRP1, which is known to be the key regulator of mitochondrial fission (Fig. 3a). However, opposite phenomenon was observed in Hep3B and SNU499 cells under enhanced expression of LL22NC03N14H11.1 (Supplementary Fig. S2A). In addition, western blot analysis presented that LL22NC03-N14H11.1 knockdown reduced, whereas its upregulation boosted S616 phosphorylation of DRP1, while the total protein level of DRP1 was not changed under the above conditions (Fig. 3b, Supplementary Fig. S2B). Previous studies proved that p-DRP1 (S616) was required for the facilitation of mitochondrial fission and also for tumor growth $^{23,26}$. Hence, these findings suggested that LL22NC03-N14H11.1 positively regulated mitochondrial fission in HCC cells through inducing p-DRP1 (S616). Later, we tried to examine whether LL22NC03-N14H11.1 influenced HCC progression through mitochondrial fission. As anticipated, it was proved that the hindered mitochondrial fission in LL22NC03-N14H11.1-silenced cells was normalized due to recovered p-DRP1 level upon DRP1 overexpression (Supplementary Fig. S2C, D). Thereafter, we confirmed that overexpression of DRP1 rescued the proliferation of HCC cells inhibited by sh- 


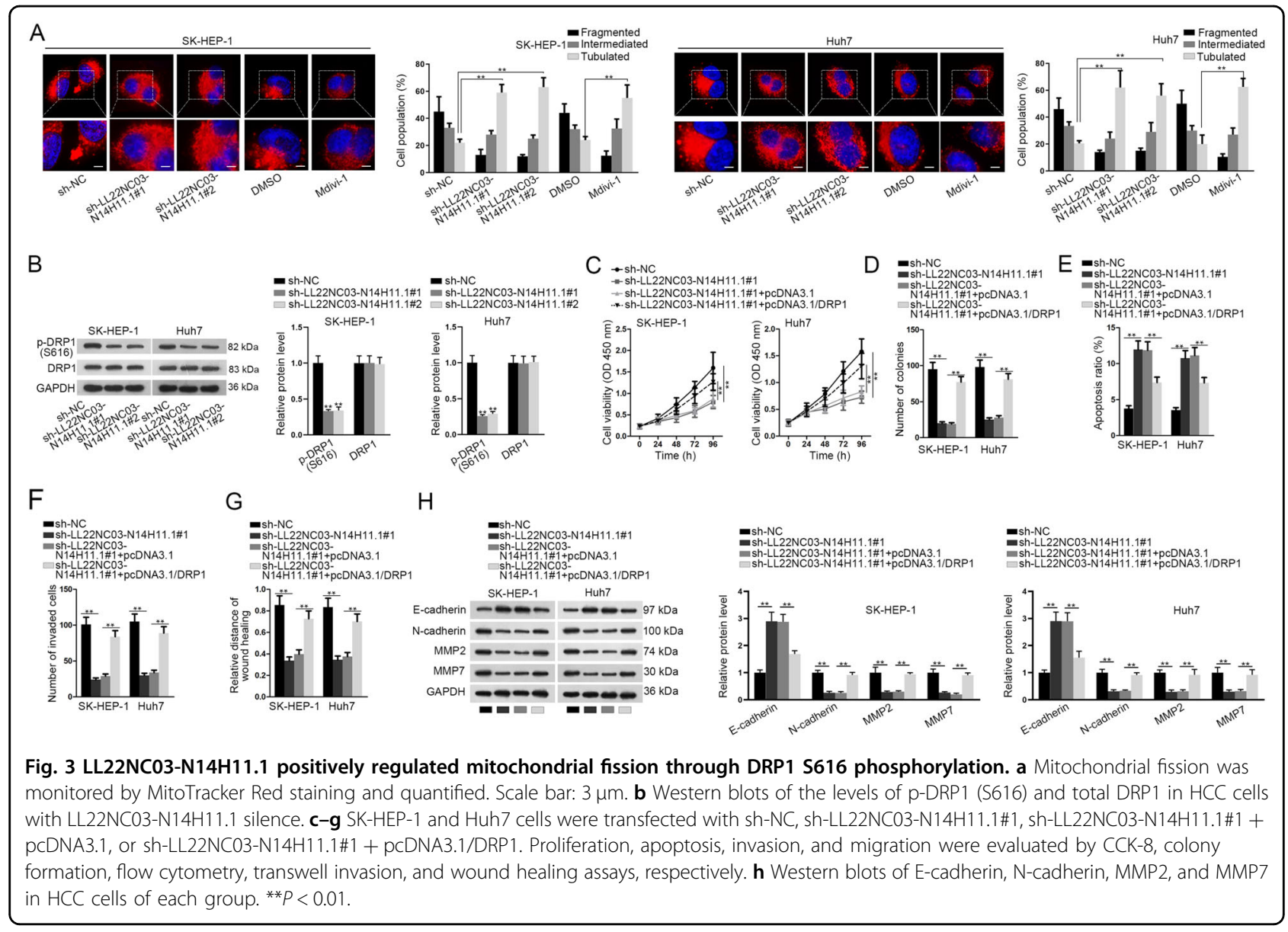

LL22NC03-N14H11.1\#1 (Fig. 3c, d). Inductive effect of sh-LL22NC03-N14H11.1\#1 on HCC cell apoptosis was abrogated by DRP1 overexpression (Fig. 3e). Invasion and migration of HCC cells restrained by sh-LL22NC03N14H11.1\#1 were restored by co-transfection of pcDNA3.1/DRP1 (Fig. 3f-g). The increase of E-cadherin and decrease of N-cadherin, MMP7, and MMP2 under LL22NC03-N14H11.1 knockdown were reversed by the overexpression of DRP1 (Fig. 3h). Jointly, it was indicated that LL22NC03-N14H11.1 promoted HCC progression through inducing p-DRP1 (S616)-facilitated mitochondrial fission.

\section{LL22NC03-N14H11.1 inhibited LZTR1-mediated ubiquitination of H-RAS (G12V) and activated MAPK pathway to induce p-DRP1 (S616)}

Thereafter, we interrogated how LL22NC03-N14H11.1 induced p-DRP (S616). Several genes that were identified to regulate $\mathrm{p}$-DRP (S616) were detected, including PDI ${ }^{23}$, CDK $1^{39}$, and ERK $1 / 2^{26}$. Western blot data illustrated that knockdown of LL22NC03-N14H11.1 resulted in no significant change in the levels of PDI1 or CDK1, but led to decreased p-ERK1/2 level, while total ERK1/2 unchanged (Fig. 4a). ERK1/2 are known to be key regulators in
MAPK pathway, and it has been reported that H-RAS (G12V) activated MAPK pathway so that ERK1/2 contributed to p-DRP (S616) and mitochondrial fission, which then drove tumor growth in pancreatic cancer ${ }^{26}$. Therefore, we detected the regulation of LL22NC03N14H11.1 on active H-RAS (G12V) in two HCC cell lines. As a result, knockdown of LL22NC03-N14H11.1 reduced the level of H-RAS (G12V) at the protein level rather than the messenger RNA (mRNA) level in HCC cells (Fig. 4b). Furthermore, we found that knockdown of LL22NC03N14H11.1 impaired the stability of H-RAS (G12V) proteins in HCC cells (Fig. 4c).

Former studies stated that LZTR1 was a regulator of ubiquitination of RAS family, and both mutant and wildtype of RAS could be ubiquitinated and degraded by $\mathrm{LZTR}^{35}$. We tried to examine whether LL22NC03N14H11.1 influenced the regulation of LZTR1 on $\mathrm{H}$ RAS (G12V). CoIP assays showed that in SK-HEP-1 cells, H-RAS (G12V) was enriched in the precipitates of antiLZTR1, and knockdown of LL22NC03-N14H11.1 enhanced such enrichment, with the input H-RAS (G12V) level decreased and LZTR1 level increased (Fig. 4d). Also, the ubiquitination of H-RAS (G12V) was induced under 


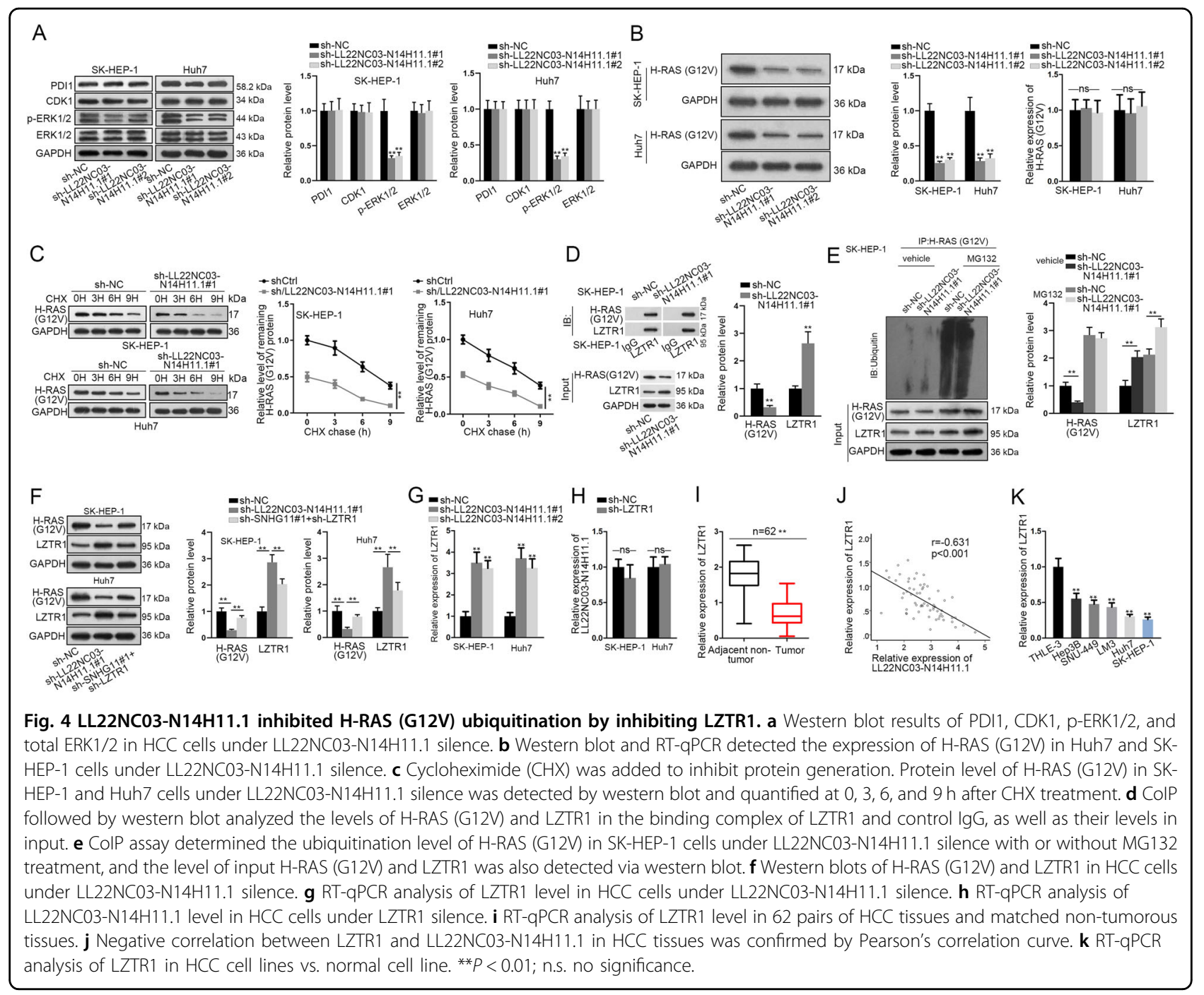

the knockdown of LL22NC03-N14H11.1, with the input HRAS (G12V) level decreased and LZTR1 level increased (Fig. 4e). These results indicated that LL22NC03-N14H11.1 reduced the interaction between LZTR1 and H-RAS (G12V) by reducing LZTR1 expression. We confirmed through western blot that knockdown of LL22NC03N14H11.1 decreased H-RAS (G12V) level and increased LZTR1 level, and such results could be reversed by the knockdown of LZTR1 in HCC cells (Fig. 4f). Additionally, silence of LL22NC03-N14H11.1A induced the mRNA level of LZTR1, but knockdown of LZTR1 had no impact on the level of LL22NC03-N14H11.1 in HCC cells (Fig. 4g, h). We then found that LZTR1 expression was downregulated and negatively correlated with LL22NC03-N14H11.1 expression in HCC tissues (Fig. 4i, j). The low expression of LZTR1 in HCC cell lines vs. normal cell line was also verified (Fig. 4k). Collectively, LL22NC03-N14H11.1 inhibited LZTR1mediated ubiquitination of H-RAS (G12V) and activated MAPK pathway to induce p-DRP1 (S616).

\section{LL22NC03-N14H11.1 transcriptionally repressed LZTR1 by recruiting $\mathrm{c}-\mathrm{Myb}$}

Subsequently, we probed the mechanism whereby LL22NC03-N14H11.1 suppressed LZTR1 expression. We found through luciferase reporter assay that the transcription activity of LZTR1 was induced under LL22NC03-N14H11.1 depletion in HCC cells (Fig. 5a). LncRNAs are reported to interact with certain transcription factors to regulate target genes $^{40,41}$. Since LL22NC03-N14H11.1 was identified to be mainly located in the nucleus of HCC cells, we deduced that LL22NC03-N14H11.1 could regulate the transcription of LZTR1 through affecting the function of certain transcription factors. We identified 65 TFs potentially targeting LZTR1 promoter from Human TFBD (http://211.67.31.242/ HumanTFDB) $(P<0.001)$ and $69 \mathrm{TFs}$ from PROMO (http:// alggen.lsi.upc.es/cgi-bin/promo_v3/promo/promoinit.cgi? $\left.\operatorname{dirDB}=\mathrm{TF} \_8.3\right) \quad(P<0.05)$. Intersection of the prediction results of two bioinformatics tools revealed 10 TFs targeting LZTR1 promoter (Fig. 5b). Pull-down analysis depicted that 


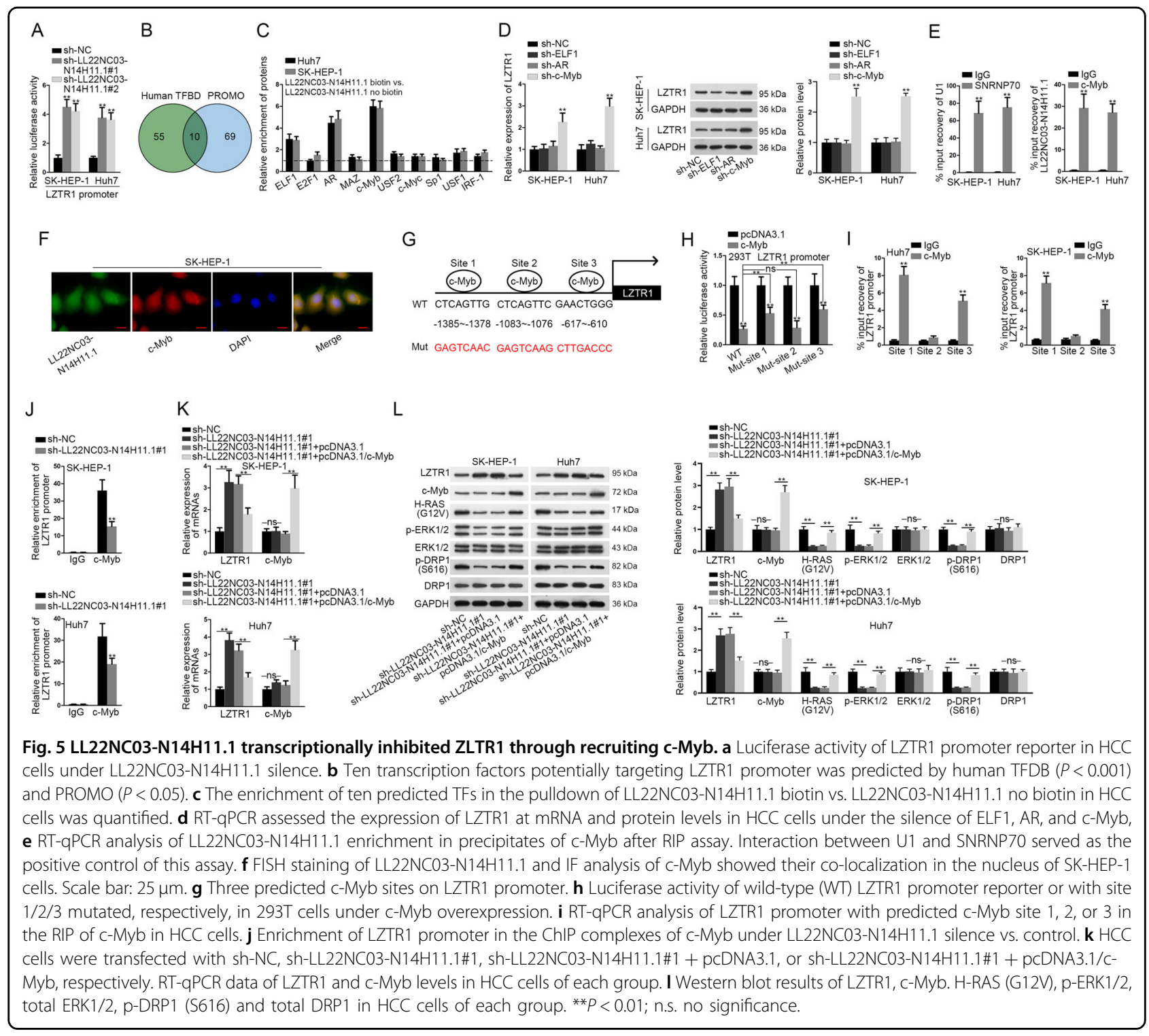

among 10 above-mentioned TFs, ELF1, AR, and c-Myb were significantly enriched in the pull-down compounds of the LL22NC03-N14H11.1 biotin group compared with the LL22NC03-N14H11.1 no biotin group (Fig. 5c). Furthermore, we observed an interesting phenomenon that the expression of LZTR1 was only induced in HCC cells after silencing c-Myb rather than other two TFs (Fig. $5 \mathrm{~d}$ ). c-Myb is a known TF that could either activate or suppress the transcription of target genes, and is involved in tumor-related activities, such as proliferation, stemness, and metastasis ${ }^{42,43}$. Recently, nuclear lncRNAs have been increasingly elucidated to recruit certain protein and serve as a scaffold to promote protein-protein or protein-RNA interactions ${ }^{44}$. Hence, we speculated that LL22NC03-N14H11.1 repressed LZTR1 transcription through recruiting c-Myb. RIP analysis confirmed the interaction between LL22NC03-N14H11.1 with
c-Myb in HCC cells (Fig. 5e). FISH and IF staining revealed the co-localization of LL22NC03-N14H11.1 and c-Myb in the nucleus of SK-HEP-1 cells (Fig. 5f). We identified three putative c-Myb sites on LZTR1 through PROMO tool (with the lowest dissimilarity) as shown in Fig. 5g. Luciferase activity of wild-type LZTR1 promoter was reduced by c-Myb overexpression and mutating site 1 or 3 partially reversed such results. However, mutating site 2 could not restore the luciferase activity of LZTR1 promoter that was inhibited by c-Myb overexpression (Fig. 5h). ChIP analysis depicted that LZTR1 promoter fragments containing site 1 and 3, rather than site 2, could be immunoprecipitated by anti-c-Myb (Fig. 5i). Moreover, knockdown of LL22NC03-N14H11.1 reduced the enrichment of LZTR1 promoter in c-Myb precipitates (Fig. 5j). In contrast, the occupancy of c-Myb on LZTR1 promoter was obviously fortified by upregulated 


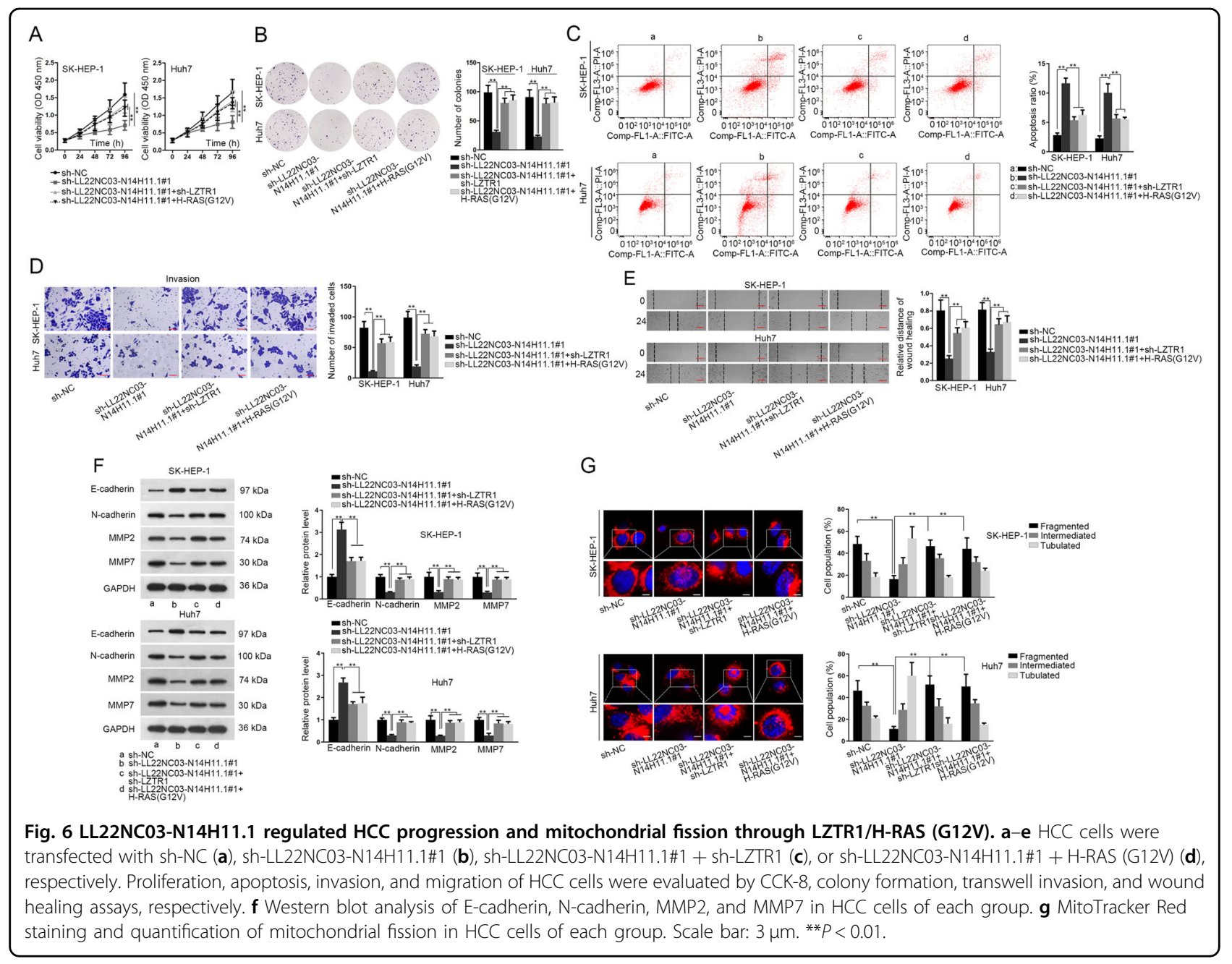

LL22NC03-N14H11.1 (Supplementary Fig. S2E). These data indicated that LL22NC03-N14H11.1 contributed to the binding of c-Myb to LZTR1 promoter. Then, we found that the level of LZTR1 induced by LL22NC03-N14H11.1 silence was reversed by c-Myb overexpression, but LL22NC03$\mathrm{N} 14 \mathrm{H} 11.1$ silence failed to influence c-Myb expression at the mRNA and protein levels (Fig. 5k, l). Also, levels of H-RAS (G12V), p-ERK1/2, and p-DRP1 (S616) reduced by shLL22NC03-N14H11.1\#1 were recovered by overexpression of c-Myb (Fig. 5l). Altogether, LL22NC03-N14H11.1 transcriptionally repressed LZTR1 through recruiting c-Myb to LZTR1 promoter, resulting in activated H-RAS/MAPK pathway.

\section{LL22NC03-N14H11.1 promoted mitochondrial fission and} HCC progression through LZTR1/H-RAS/MAPK pathway

To detect whether LL22NC03-N14H11.1 regulated HCC progression and mitochondrial fission through LZTR1/HRAS/MAPK pathway, we designed rescue experiments. As a result, either LZTR1 knockdown or H-RAS (G12V) overexpression rescued proliferation of $\mathrm{HCC}$ cells with
LL22NC03-N14H11.1 silence (Fig. 6a, b). Apoptosis of HCC cells increased by LL22NC03-N14H11.1 knockdown was reversed by LZTR1 silence or H-RAS (G12V) overexpression (Fig. 6c). Inhibitory effect of LL22NC03$\mathrm{N} 14 \mathrm{H} 11.1$ depletion on HCC cell invasion and migration was abrogated by silencing LZTR1 or overexpressing $\mathrm{H}$ RAS (G12V) (Fig. 6d, e). LZTR1 depletion or H-RAS (G12V) overexpression reversed the increase of E-cadherin and decrease of $\mathrm{N}$-cadherin, MMP2, and MMP7 in LL22NC03-N14H11.1-silenced HCC cells (Fig. 6f). The attenuated mitochondrial fission under LL22NC03$\mathrm{N} 14 \mathrm{H} 11.1$ silence in HCC cells was restored by the knockdown of LZTR1 or overexpression of H-RAS (G12V) (Fig. 6g). Altogether, LL22NC03-N14H11.1 aggravated malignant phenotypes in HCC through targeting LZTR1/ H-RAS/MAPK pathway.

\section{LL22NC03-N14H11.1 facilitated tumor growth and metastasis of $\mathrm{HCC}$ in vivo}

Finally, in vivo assays were conducted to confirm the role of LL22NC03-N14H11.1 in HCC. SK-HEP-1 cells 
were transfected with sh-NC, sh-LL22NC03-N14H11.1\#1, or sh-LL22NC03-N14H11.1\#1 + sh-LZTR1, respectively, and subcutaneously injected into nude mice to establish xenografts. Consequently, LL22NC03-N14H11.1 knockdown retarded tumor growth of HCC in vivo, but cotransfection of sh-LZTR1 abrogated such effect (Fig. 7a). LL22NC03-N14H11.1 depletion reduced tumor weight, and co-transfection of sh-LZTR1 restored such reduction (Fig. 7b). The apoptosis of HCC cells induced by shLL22NC03-N14H11.1\#1 in vivo was impaired by LZTR1 silence (Fig. 7c). RT-qPCR data confirmed that knockdown of LL22NC03-N14H11.1 reduced LL22NC03-N14H11.1 level and induced LZTR1 level, and co-transfection of sh-LZTR1 impaired the induction of LZTR1 level, but had no influence on LL22NC03N14H11.1 expression (Fig. 7d). Western blot analysis verified that LL22NC03-N14H11.1 knockdown induced LZTR1, but decreased H-RAS (G12V), p-ERK1/2, and pDRP1 (S616) in vivo, and such results were reversed by LZTR1 silence, with c-Myb, total ERK1/2, and total DRP1 levels unchanged all the way (Fig. 7e). IHC staining exhibited that the positivity of proliferation index (Ki67 and PCNA) and mesenchymal marker (N-cadherin) was reduced, and epithelial marker (E-cadherin) was induced in xenografts with LL22NC03-N14H11.1 silence, while knockdown of LZTR1 reversed these results (Fig. $7 f-g$ ). HE staining revealed that the number of in vivo metastatic nodules was lessened by LL22NC03-N14H11.1 knockdown, but recovered by the silence of LZTR1 (Fig. 7h). In conclusion, it was suggested that LL22NC03-N14H11.1 facilitated tumor growth and metastasis of HCC through LZTR1 in vivo.

\section{Discussion}

Studies have provided convincing evidences that hepatocarcinogenesis involves dysregulation of lncRNAs. Large amounts of lncRNAs are identified to possess prognostic and diagnostic values in $\mathrm{HCC}^{8,9}$. Therefore, identifying novel lncRNAs in $\mathrm{HCC}$ and finding out their modulatory mechanism can benefit the progress of treatment efficacy in HCC and improve the survival of HCC patients.

This study analyzed the dysregulated lncRNAs in HCC through two bioinformatics tools (circlncRNAnet and GEPIA) and found that LL22NC03-N14H11.1 was a new lncRNA highly expressed in HCC samples and had great prognostic significance for HCC patients. We also confirmed that LL22NC03-N14H11.1 level was elevated in HCC tissues and cell lines. Besides, we found that LINC00152 was also highly expressed in HCC samples. A number of former studies have elucidated that LINC00152 served as an oncogene in $\mathrm{HCC}^{36-38}$, but the role of LL22NC03-N14H11.1 in HCC has never been investigated. Therefore, we focused on the exploration of
LL22NC03-N14H11.1 in HCC. Functionally, we discovered that LL22NC03-N14H11.1 accelerated proliferation, migration, invasion, and EMT in HCC cells. These findings indicated that targeting LL22NC03-N14H11.1 in HCC might be a promising approach to relieve hepatocarcinogenesis.

Mitochondrial fission has been demonstrated as an important activity related to cell survival and metastasis in tumors. Some works stated that increased mitochondrial fission contributes to drug-induced apoptosis and enhance chemo-sensitivity in cancer cells ${ }^{45}$, whereas others argued that inhibiting mitochondrial fission can retard cell proliferation and metastasis in several cancer types, such as colon cancer, lung cancer, and breast cancer $^{14-18}$. A recent study observed that mitochondrial fission was facilitated in HCC samples compared to adjacent normal samples, and that DRP1 inhibition could inhibit cell survival and autophagy in $\mathrm{HCC}^{19}$, indicating that excessive mitochondrial fission in $\mathrm{HCC}$ was oncogenic. In this study, we found that LL22NC03N14H11.1 silence prevented mitochondrial fission, same as the effect of Midivi-1 (DRP1 inhibitor). Also, we validated that overexpressing DRP1 could rescue the inhibitory effect of LL22NC03-N14H11.1 silence on HCC progression, indicating that LL22NC03-N14H11.1 regulated HCC through facilitating DRP1-regulated mitochondrial fission.

DRP1 is a well-known member of dynamin-related GTPases, which were referred to as primary regulators of mitochondrial dynamics ${ }^{13,20}$. It has been well established that phosphorylation of DRP1 at S616 led to DRP1 activation and facilitated mitochondrial fission ${ }^{21}$. Herein, we found that LL22NC03-N14H11.1 contributed to DRP1 phosphorylation at S616, with no impact on the level of total DRP1. Previous works have identified several regulators of p-DRP1 (S616), such as CDK1, DPI1, and ERK $1 / 2^{22-24}$. Herein, we discovered that among the aforementioned regulators, only ERK1/2 phosphorylation was positively regulated by LL22NC03-N14H11.1 in HCC cells. ERK1/2 was the key regulators in ERK/MAPK pathway. It is widely known that MAPK pathway is a key signaling related to the survival, apoptosis, and metastasis in tumor cells ${ }^{46,47}$. Recent studies revealed that H-RAS (G12V) activated MAPK pathway so that ERK1/2 was phosphorylated and induced p-DRP1 $(\mathrm{S} 616)^{25}$. Importantly, such mechanism has been proved to accelerate mitochondrial fission and tumor growth in pancreatic cancer $^{26}$. Hence, it was reasonable to deduce that LL22NC03-N14H11.1 regulated MAPK pathway to induce mitochondrial fission in HCC.

Expectedly, we firstly found that LL22NC03-N14H11.1 knockdown reduced H-RAS (G12V) level in HCC cells. We further suggested that LL22NC03-N14H11.1 regulated H-RAS (G12V) protein stability. Several studies have 


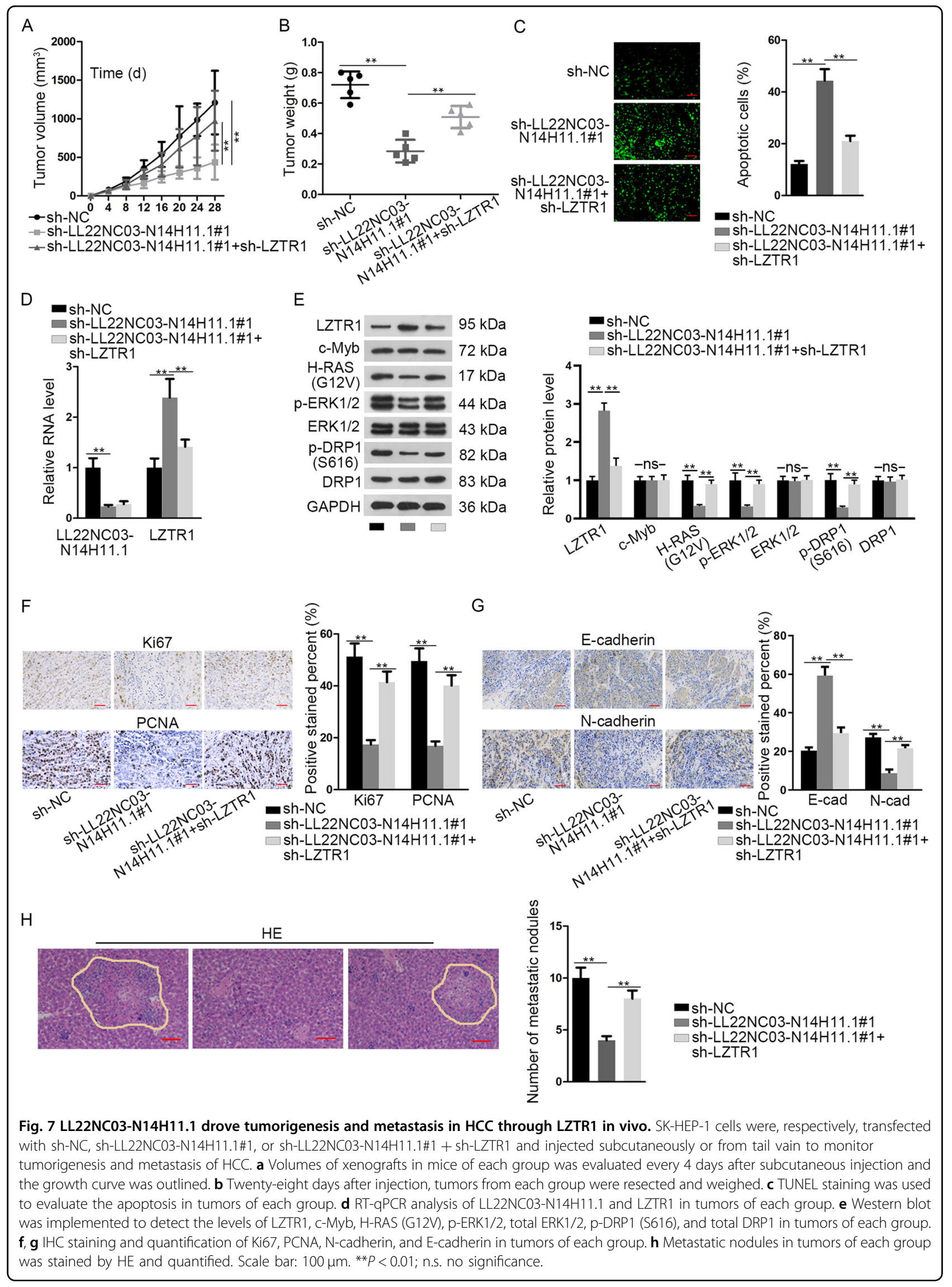




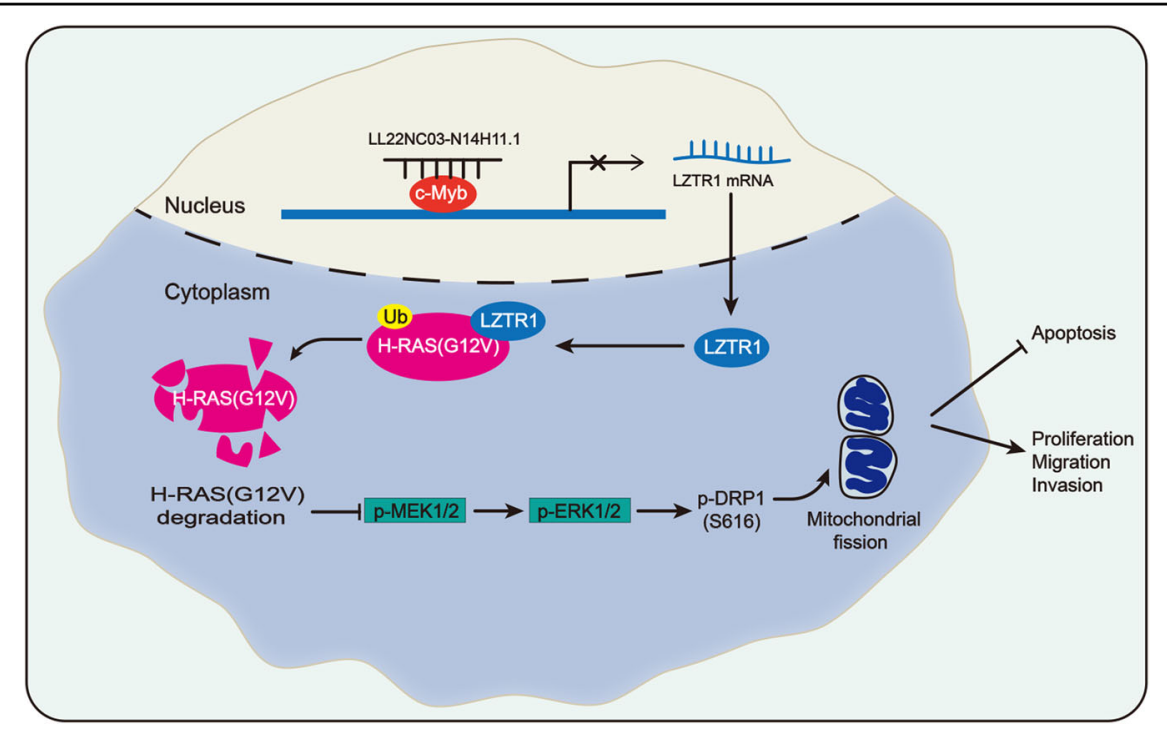

Fig. 8 Graphical presentation of LL22NC03-N14H11.1/c-Myb/LTZR1/H-RAS/MAPK/DRP1 axis in HCC. LL22NC03-N14H11.1 interacted with c-Myb to transcriptionally repress LZTR1, so as to decrease H-RAS (G12V) ubiquitination, activate MAPK pathway, and induce p-DRP1 (S616), resulting in induced mitochondrial fission and accelerated HCC progression.

pointed out that LZTR1 was a conserved regulator of the ubiquitination of RAS family. LZTR1 was suggested to possess tumor-suppressing function in cancers and be related to cell apoptosis ${ }^{32-34}$. A study elucidated that LZTR1 ubiquitinated K-RAS, M-RAS, N-RAS, H-RAS, and their mutant types, leading to the inactivation of MAPK pathway ${ }^{35}$. Therefore, it was reasonable to suggest that LZTR1 exerted tumor-suppressive effect through suppressing MAPK pathway, and that LZTR1 might mediate the regulation of LL22NC03-N14H11.1 on HRAS (G12V) in HCC. This study firstly revealed that LL22NC03-N14H11.1 reduced LZTR1 expression to inhibit the LZTR1-mediated ubiquitination of H-RAS (G12V).

Furthermore, we uncovered that LL22NC03-N14H11.1 repressed LZTR1 expression at the transcriptional level. We identified that LL22NC03-N14H11.1 was mainly located in the nucleus of HCC cells. The mechanisms whereby lncRNAs regulate target genes are different depending on their cellular localization ${ }^{10,11}$. In the nucleus, IncRNAs can recruit certain transcription factors to affect the transcription of target genes ${ }^{40,41}$. For instance, lncRNA REG1CP could tether FANCJ to REG3A promoter to induce REG3A activation by interacting with FANCJ in colorectal cancer ${ }^{48}$. Also, a latest research discovered that IncRNA Oplr16 could activate Oct4 via inducing DNA demethylation by recruiting $\mathrm{TET} 2^{49}$. Herein, we identified that c-Myb potentially targeted LZTR1 promoter and interacted with LL22NC03-N14H11.1. As a transcription factor, c-Myb is demonstrated to either suppress or activate gene transcription $^{42,43}$, and it has been proved to contribute to hepatocarcinogenesis and metastasis in $\mathrm{HCC}^{50}$. In this study, we validated that LL22NC03-N14H11.1 recruited c-Myb to LZTR1 promoter so as to repress LZTR1 transcription and activate H-RAS/MAPK pathway, suggesting LL22NC03-N14H11.1 as a scaffold in HCC as many other lncRNAs do ${ }^{44,51}$. Besides, it has been revealed that c-Myb could repress transcription by recruiting certain transcription repressor or competing with other transcription activators for target promoters ${ }^{43}$. However, the precise mechanism whereby c-Myb inhibited LZTR1 transcription remains yet to be further investigated in the future. Finally, we validated that LL22NC03-N14H11.1 promoted HCC tumorigenesis and metastasis in vivo.

\section{Conclusions}

This study firstly revealed a novel lncRNA LL22NC03$\mathrm{N} 14 \mathrm{H} 11.1$ as an oncogene in HCC. Functionally, LL22NC03-N14H11.1 promoted proliferation, invasion, migration, and prevented apoptosis in vitro and drove tumorigenesis and metastasis in vivo. Besides, LL22NC03N14H11.1 promoted p-DRP1 (S616) to facilitate mitochondrial fission. Mechanistically, LL22NC03-N14H11.1 interacted with c-Myb to transcriptionally repress LZTR1, so as to decrease H-RAS (G12V) ubiquitination, activate MAPK pathway, and induce p-DRP1 (S616) (Fig. 8). These findings indicated LL22NC03-N14H11.1 as a new target in $\mathrm{HCC}$ and might provide new thoughts for the lncRNA-targeted molecular therapy in HCC.

\section{Acknowledgements}

We thank all the members involved in this study. The study was supported by Guangxi Clinic Medicine Research Center of Hepatobiliary Diseasea (No. AD17129025). 


\section{Author details}

'Gastrointestinal Medicine, Affiliated Hospital of YouJiang Medical University For Nationalities, Baise, Guangxi 533000, P. R. China. 'Laboratory Medicine, Affiliated Hospital of YouJiang Medical University For Nationalities, Baise, Guangxi 533000, P. R. China. ${ }^{3}$ Blood transfusion Department, Affiliated Hospital of YouJiang Medical University For Nationalities, Baise, Guangxi 533000, P. R. China

\section{Conflict of interest}

The authors declare that they have no conflict of interest.

\section{Publisher's note}

Springer Nature remains neutral with regard to jurisdictional claims in published maps and institutional affiliations.

Supplementary Information accompanies this paper at (https://doi.org/ 10.1038/s41419-020-2584-z)

Received: 29 October 2019 Revised: 20 April 2020 Accepted: 20 April 2020 Published online: 07 October 2020

\section{References}

1. Bosch, F. X., Ribes, J., Cléries, R. \& Díaz, M. Epidemiology of hepatocellular carcinoma. Clin. Liver Dis. 9, 191-211 (2005).

2. Llovet, J. M. et al. Hepatocellular carcinoma. Nat. Rev. Dis. Prim. 2, 16018 (2016).

3. Bertuccio, P. et al. Global trends and predictions in hepatocellular carcinoma mortality. J. Hepatol. 67, 302-309 (2017).

4. Cheetham, S. W., Gruhl, F., Mattick, J. S. \& Dinger, M. E. Long noncoding RNAs and the genetics of cancer. Br. J. Cancer 108, 2419-2425 (2013).

5. Flintoft, L. Structure and function for IncRNAs. Nat. Rev. Genet. 14, 598 (2013).

6. Chen, D. L. et al. Long non-coding RNA UICLM promotes colorectal cancer liver metastasis by acting as a ceRNA for microRNA-215 to regulate ZEB2 expression. Theranostics 7, 4836-4849 (2017).

7. He, W. et al. MSC-regulated IncRNA MACC1-AS1 promotes stemness and chemoresistance through fatty acid oxidation in gastric cancer. Oncogene $\mathbf{3 8}$ 4637-4654 (2019)

8. Wang, S. et al. NEAT1 paraspeckle promotes human hepatocellular carcinoma progression by strengthening IL-6/STAT3 signaling. Oncoimmunology 7, e1503913-e1503913 (2018)

9. Li, Z. et al. The LINC01138 drives malignancies via activating arginine methyltransferase 5 in hepatocellular carcinoma. Nat. Commun. 9, 1572-1572 (2018)

10. Dong, $H$. et al. SP1-induced IncRNA AGAP2-AS1 expression promotes chemoresistance of breast cancer by epigenetic regulation of MyD88. J. Exp. Clin. Cancer Res. 37, 202-202 (2018).

11. Lin, C. et al. Transcriptional and posttranscriptional regulation of HOXA13 by IncRNA HOTTIP facilitates tumorigenesis and metastasis in esophageal squamous carcinoma cells. Oncogene 36, 5392 (2017).

12. Marchese, F. P., Raimondi, I. \& Huarte, M. The multidimensional mechanisms of long noncoding RNA function. Genome Biol. IF11. 908 18, 206 (2017).

13. Westermann, B. Mitochondrial fusion and fission in cell life and death. Nat. Rev. Mol. Cell. Biol. 11, 872 (2010)

14. Inoue-Yamauchi, A. \& Oda, H. Depletion of mitochondrial fission factor DRP1 causes increased apoptosis in human colon cancer cells. Biochem. Biophys. Res. Commun. 421, 81-85 (2012)

15. Arismendi-Morillo, G. Electron microscopy morphology of the mitochondrial network in human cancer. Int. J. Biochem. Cell Biol. 41, 2062-2068 (2009).

16. Rehman, J. et al. Inhibition of mitochondrial fission prevents cell cycle progression in lung cancer. FASEB J. 26, 2175-2186 (2012).

17. Zhao, J. et al. Mitochondrial dynamics regulates migration and invasion of breast cancer cells. Oncogene 32, 4814-4824 (2013).

18. Zhang, Y. et al. MTP18 overexpression contributes to tumor growth and metastasis and associates with poor survival in hepatocellular carcinoma. Cell Death Dis. 9, 956 (2018).

19. Huang, Q. et al. Increased mitochondrial fission promotes autophagy and hepatocellular carcinoma cell survival through the ROS-modulated coordinated regulation of the NFKB and TP53 pathways. Autophagy $\mathbf{1 2}$ 999-1014 (2016).

20. Smirnova, E., Griparic, L., Shurland, D. L. \& van der Bliek, A. M. Dynamin-related protein Drp1 is required for mitochondrial division in mammalian cells. Mol. Biol. Cell 12, 2245-2256 (2001)

21. Cook, S. J., Stuart, K. Gilley, R. \& Sale, M. J. Control of cell death and mitochondrial fission by ERK1/2 MAP kinase signalling. FEBS J. 284, 4177-4195 (2017).

22. Taguchi, N., Ishihara, N., Jofuku, A., Oka, T. \& Mihara, K. Mitotic phosphorylation of dynamin-related GTPase Drp1 participates in mitochondrial fission. J. Biol. Chem. 282, 11521-11529 (2007).

23. Lee, D. S. \& Kim, J. E. PDI-mediated S-nitrosylation of DRP1 facilitates DRP1S616 phosphorylation and mitochondrial fission in CA1 neurons. Cell Death Dis. 9, 869 (2018)

24. $\mathrm{Yu}, \mathrm{T}$., Jhun, B. S. \& Yoon, Y. High-glucose stimulation increases reactive oxygen species production through the calcium and mitogen-activated protein kinase-mediated activation of mitochondrial fission. Antioxid. Redox Signal. 14, 425-437 (2011).

25. Serasinghe, M. N. et al. Mitochondrial division is requisite to RAS-induced transformation and targeted by oncogenic MAPK pathway inhibitors. Mol. Cell 57, 521-536 (2015).

26. Kashatus, J. A. et al. Erk2 phosphorylation of Drp1 promotes mitochondrial fission and MAPK-driven tumor growth. Mol. Cell 57, 537-551 (2015).

27. Nacak, T. G., Leptien, K., Fellner, D., Augustin, H. G. \& Kroll, J. The BTB-kelch protein LZTR-1 is a novel Golgi protein that is degraded upon induction of apoptosis. J. Biol. Chem. 281, 5065-5071 (2006).

28. Geyer, R., Wee, S., Anderson, S., Yates, J. \& Wolf, D. A. BTB/POZ domain proteins are putative substrate adaptors for cullin 3 ubiquitin ligases. Mol. Cell 12 783-790 (2003).

29. Furukawa, M., He, Y. J., Borchers, C. \& Xiong, Y. Targeting of protein ubiquitination by BTB-Cullin 3-Roc1 ubiquitin ligases. Nat. Cell Biol. 5, 1001-1007 (2003).

30. Stogios, P. J. \& Privé, G. G. The BACK domain in BTB-kelch proteins. Trends Biochem. Sci. 29, 634-637 (2004)

31. Lu, A. \& Pfeffer, S. R. A CULLINary ride across the secretory pathway: more than just secretion. Trends Cell Biol. 24, 389-399 (2014).

32. Piotrowski, A. et al. Germline loss-of-function mutations in LZTR1 predispose to an inherited disorder of multiple schwannomas. Nat. Genet. 46, 182-187 (2014).

33. Frattini, $V$. et al. The integrated landscape of driver genomic alterations in glioblastoma. Nat. Genet. 45, 1141-1149 (2013).

34. Franceschi, S. et al. Molecular portrait of a rare case of metastatic glioblastoma: somatic and germline mutations using whole-exome sequencing. NeuroOncology 18, 298-300 (2016).

35. Abe, T. et al. LZTR1 facilitates polyubiquitination and degradation of RASGTPases. Cell Death Differ. https://doi.org/10.1038/s41418-019-0395-5 (2019).

36. Wang, J. et al. Insight into the molecular mechanism of LINC00152/miR-215/ CDK13 axis in hepatocellular carcinoma progression. J. Cell. Biochem. https:// doi.org/10.1002/jcb.29197 (2019)

37. Ma, P. et al. LINC00152 promotes cell cycle progression in hepatocellular carcinoma via miR-193a/b-3p/CCND1 axis. Cell Cycle (Georget., Tex.) 17 974-984 (2018).

38. Deng, $\mathbf{X}$. et al. Linc00152 promotes cancer progression in hepatitis B virusassociated hepatocellular carcinoma. Biomed. Pharmacother. 90, 100-108 (2017).

39. Kashatus, D. F. et al. RALA and RALBP1 regulate mitochondrial fission at mitosis. Nat. Cell Biol. 13, 1108-1115 (2011).

40. Lei, J. J. et al. Long noncoding RNA CDKN2B-AS1 interacts with transcription factor BCL11A to regulate progression of cerebral infarction through mediating MAP4K1 transcription. FASEB J. https://doi.org/10.1096/fj.201802252R (2019).

41. Long, Y., Wang, X., Youmans, D. T. \& Cech, T. R. How do IncRNAs regulate transcription? Sci. Adv. 3, eaao2110-eaao2110 (2017)

42. Li, Y. et al. c-Myb enhances breast cancer invasion and metastasis through the Wnt/ß-catenin/Axin2 pathway. Cancer Res. https://doi.org/10.1158/0008-5472. can-15-2302 (2016).

43. Gautam, S. et al. The transcription factor c-Myb regulates CD8+ T cell stemness and antitumor immunity. Nat. Immunol. 20, 337-349 (2019).

44. Wang, Y. et al. The IncRNA PVT1 regulates nasopharyngeal carcinoma cell proliferation via activating the KAT2A acetyltransferase and stabilizing HIF-1a Cell Death Differ. 27, 695-710 (2020). 
45. Tian, T. et al. Long noncoding RNA MPRL promotes mitochondrial fission and cisplatin chemosensitivity via disruption of pre-miRNA processing. Clin. Cancer Res. https://doi.org/10.1158/1078-0432.ccr-18-2739 (2019).

46. Ma, M. et al. MicroRNA-23a-3p inhibits mucosal melanoma growth and progression through targeting adenylate cyclase 1 and attenuating CAMP and MAPK pathways. Theranostics 9, 945-960 (2019).

47. Chen, J. et al. Human mesenchymal stem cells promote tumor growth via MAPK pathway and metastasis by epithelial mesenchymal transition and integrin a5 in hepatocellular carcinoma. Cell Death Dis. 10, 425 (2019).

48. Yari, H. et al. LnCRNA REG1CP promotes tumorigenesis through an enhancer complex to recruit FANCJ helicase for REG3A transcription. Nat. Commun. 10 5334-5334 (2019).
49. Jia, L. et al. Oplr16 serves as a novel chromatin factor to control stem cell fate by modulating pluripotency-specific chromosomal looping and TET2-mediated DNA demethylation. Nucleic Acids Res. 48, 3935-3948 (2020)

50. Lan, T. et al. LnCRNA SNHG10 facilitates hepatocarcinogenesis and metastasis by modulating its homolog SCARNA13 via a positive feedback loop. Cancer Res. https://doi.org/10.1158/0008-5472.can-18-4044 (2019).

51. Chen, $X$. et al. Long noncoding RNA $L B C S$ inhibits self-renewal and chemoresistance of bladder cancer stem cells through epigenetic silencing of SOX2. Clin. Cancer Res. 25, 1389-1403 (2019). 